\title{
A Mobile Anchor Node Positioning Algorithm for WSN in Three-dimensional Space
}

\author{
Xiaochao Dang ${ }^{1, a}$, Fenfang $\mathrm{Li}^{2, b}$,Zhanjun $\mathrm{Hao}^{3, \mathrm{c}}$ (Contact author) \\ ${ }^{1}$ College of Computer Science and Engineering, Northwest Normal University, Lanzhou 730070, \\ P.R. China \\ Gansu IOT Research Center, Lanzhou 730070, P.R. China \\ ${ }^{2}$ College of Computer Science and Engineering, Northwest Normal University, Lanzhou 730070, P.R. \\ China \\ ${ }^{3}$ College of Computer Science and Engineering, Northwest Normal University, Lanzhou 730070, \\ P.R. China \\ Gansu IOT Research Center, Lanzhou 730070, P.R. China \\ a1246531647@qq.com, 'PImuziff@126.com, 'zhanjunhao@126.com
}

\begin{abstract}
Keywords: wireless sensor network; node localization; mobile anchor node; static anchor node; fuzzy information; localization accuracy.

Abstract. In wireless sensor network, in order to effectively reduce the influence of the complex environment in node localization accuracy, based on trilateral plane positioning algorithm, this paper proposes an algorithm about the mobile anchor node and fuzzy information (Mobile Anchor Node Localization on Fuzzy Information, referred to as MANLFI) to achieve a node localization in three-dimensional space. By constantly updating the speed and position of the anchor node, we measure the direction angle and pitch angle between anchor nodes and other nodes to achieve node localization. In this paper, when nodes are located by algorithm, which will act as static anchor nodes to attach other nodes. Experiment shows that, compared with APIT-3D, the bounding cube algorithm of MANLFI improves the localization accuracy of nodes and the stability of network and with less delay.
\end{abstract}

\section{Introduction}

Wireless sensor network ${ }^{[1-3]}$ (referred to as WSN) is a promising technology at present, with the continuous advancement of technology, the demand for the application of WSN is increasing. Node localization is one of the supporting technologies of WSN. If the node position is unknown, the monitoring information of sensor node will make no sense, and do not work efficiently, that is, the high performance of network cannot be achieved. WSN usually demands a large amount of distributed computing while nodes are relatively simple. For node's performance is limited by energy $^{[4-5]}$,volume and the network cost, WSN is only equipped with GPS equipment on a smart part of nodes to obtain their positional coordinates which called anchor nodes. Most nodes must obtain their locational information through special positioning algorithm. As well as constrained by nodes hardware volume, functionality, and power consumption, many classic positioning algorithms applied in three-dimensional space are hard. Therefore, the low-power consumption with high-accuracy ${ }^{8}$ positioning algorithm for WSN is a hot issue at present ${ }^{[9-11]}$. Currently, the positioning algorithm for WSN is mainly focused on two-dimensional space, just a little number of algorithms are dealing with three-dimensional space ${ }^{[12-14]}$.

Traditional positioning algorithm models are all concentrating on centroid. The realization of these algorithms is to calculate the geometric centroid of the anchor nodes among the range of node communication with its own position. Specifically speaking, anchor nodes will broadcast its own ID and coordinate information signal to their neighbor nodes ${ }^{[15-17]}$. When the unknown node monitors the number of signal which is larger than the threshold value preseted, the geometric centroid of the polygon maked up with these anchor nodes within the communication range is defined as the location of the unknown node. The algorithm in this paper is not based on centroid algorithm. The 
final coordinate is determined by computing the several possible nodes coordinates to improve the positioning accuracy and the coverage $\mathrm{e}^{[18-19]}$ of space.

The passive sensor network only provides angular measurement, and the destination coordinates of node can be determined by the cross-sight that observed simultaneously by several sensor nodes or a single mobile anchor node ${ }^{[20-22]}$. These methods are based on the triangulation measurements in the condition of none measurement errors to uniquely identify the intersection of the line of sight. In this paper, we present an algorithm called MANLFI that of analyzing the passive target mobile positioning algorithm based on fuzzy theory ${ }^{[23-25]}$. By repeatedly measuring the direction angle and pitch angle of the passive target to realize the geometric positioning algorithm in three-dimensional space. Algorithm presented in this paper needs pre-deployment ${ }^{[26-27]}$ of some anchor nodes. Based on fuzzy geometry theory, the current fuzzy positioning algorithm is to achieve node localization with useing anchor node's fuzzy information, including direction angle, pitch angle to locate the nodes in three-dimensional space, and constantly update the speed ${ }^{[28-29]}$, direction, and position of the anchor node after location.

\section{A Method about Node's Fuzzy Information Positioning Based on Mobile Anchor in Three-Dimensional Space (MANLFI)}

Define $\bar{P}_{i}\left(X_{i}, y_{i}, z_{i}\right)$ is the sensor node fuzzy observing position of plane. The fuzzy direction angle is $\overline{\theta_{k}}$, the blurred pitch angle is $\overline{\varphi_{k}}$ and the position of the target node is $\bar{P}(x, y, z)$, using some nodes that deployed in plane to locate other unknown nodes, then the node's $z, y$ coordinates can be calculated by fuzzy geometric positioning algorithm. Cross the line of sight targeting methods which show that these anchor nodes carry some information such as the fuzzy direction angle and pitch angle with the unknown nodes. Using the nodes' positions information and its coordinate has been determined by the fuzzy direction angle and pitch angle as the node target information. Fig.1 is an example of the direction angle and pitch angle with two anchor nodes. $\left(\overline{\theta_{i}}, \overline{\varphi_{i}}\right)$ and $\left(\overline{\theta_{j}}, \overline{\varphi_{j}}\right)$ are the observations sights of an anchor node.

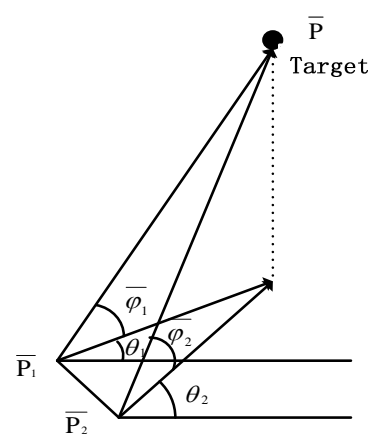

Fig.1. Geometric positioning fuzzy information of two sensor nodes

Using the angle Eq.1 in three-dimensional space to calculate the fuzzy error:

$e_{k}: e_{k}=S_{k} \sin \beta$

Specific calculating means following from Eq.2 to Eq.5:

Min $e=\sum_{k} W_{k} e_{k}$

$\beta=\cos ^{-1}\left(\frac{l_{1} l_{2}+m_{1} m_{2}+n_{1} n_{2}}{\sqrt{l_{1}^{2}+m_{1}^{2}+n_{1}^{2}}+\sqrt{l_{2}^{2}+m_{2}^{2}+n_{2}^{2}}}\right)$

In Eq.3,

$l_{1}=\cos \left(\varphi_{k}\right) \cdot \cos \left(\theta_{k}\right), m_{1}=\cos \left(\varphi_{k}\right) \cdot \sin \left(\theta_{k}\right), n_{1}=\sin \left(\varphi_{k}\right)$ 


$$
\begin{aligned}
& l_{2}=\cos \left(\overline{\varphi_{k}}\right) \cdot \cos \left(\overline{\theta_{k}}\right), m_{2}=\cos \left(\overline{\varphi_{k}}\right) \cdot \sin \left(\overline{\theta_{k}}\right), n_{2}=\sin \left(\overline{\varphi_{k}}\right) \\
& h_{i}=d\left(P_{i}, P_{j}\right) \cdot \tan \left(\overline{\varphi_{i}}\right), h_{j}=d\left(P_{i}, P_{j}\right) \cdot \tan \left(\overline{\varphi_{j}}\right) \\
& x=\bar{X}_{i}+d\left(P_{i}, P_{j}\right) \cdot \cos \left(\bar{\theta}_{i}\right), y=\bar{y}_{i}+d\left(P_{i}, P_{j}\right) \cdot \sin \left(\overline{\theta_{i}}\right), z=\bar{Z}_{i}+\frac{h_{i}+h_{j}}{2}
\end{aligned}
$$

In Eq.4 and Eq.5, $i=1, \ldots, N-1, j=i+1, \ldots, N, k=1,2, \ldots N, S_{k}$ is the distance between the fuzzy node $\overline{P_{k}}$ and the target node $\bar{P}$.

The weighted calculation formula can be used to update the node's position. Assume a node named $P(x, y, z)$ which has n-possible coordinates, i.e.. $P_{1}\left(x_{1}, y_{1}, z_{1}\right), P_{2}\left(x_{2}, y_{2}, z_{2}\right), \ldots, P_{n}\left(x_{n}, y_{n}, z_{n}\right) . \bar{x}$ is the average value in $x$ direction of $P(x, y, z), \bar{y}$ is the average value in y direction of $P(x, y, z), \bar{z}$ is the average value in $z$ direction of $P(x, y, z)$, and described as Eq.6:

$$
\begin{gathered}
\bar{x}=\frac{1}{n} \sum_{i=1}^{n} X_{i}, \bar{y}=\frac{1}{n} \sum_{i=1}^{n} y_{i}, \bar{z}=\frac{1}{n} \sum_{i=1}^{n} Z_{i} \\
X=\frac{\sum_{i=1}^{n} \frac{X_{i}}{X}}{\sum_{i=1}^{n} \frac{1}{X_{i}}}, y=\frac{\sum_{i=1}^{n} \frac{y_{i}}{\bar{y}}}{\sum_{i=1}^{n} \frac{1}{X_{i}}}, z=\frac{\sum_{i=1}^{n} \frac{Z_{i}}{Z}}{\sum_{i=1}^{n} \frac{1}{Z_{i}}}
\end{gathered}
$$

Eq.7 is the weighting Equation, through these steps we obtained the latest coordinate $(x, y, z)$ of the unknown node.

After each round of the algorithm, when the distance between anchor nodes is not within the above mentioned range in three-dimensional space, then the speed and direction of the anchor nodes will be updated by Eq.8:

$$
v_{k}=\eta V_{k-1}+(1-\eta) \bar{v}, \quad d_{k}=\eta d_{k-1}+(1-\eta) \bar{d}
$$

In Eq.8, $\boldsymbol{v}_{k}$ and $\boldsymbol{d}_{k}$ are the speed and direction of anchor node in $\mathrm{k}$ moment, $\bar{v}$ and $\bar{d}$ are the average speed and direction of anchor node, $\eta$ is a random parameter and its range is in $0 \leq \eta \leq 1$. When the speed and direction of anchor node are confirmed, the new position of the anchor node can be calculated by Eq.9:

$$
X_{k+1}=X_{k}+V_{k} \cdot\left(\cos \left(\frac{d_{k}}{2}\right)\right)^{2}, y_{k+1}=y_{k}+V_{k} \cdot\left(\sin \left(\frac{d_{k}}{2}\right)\right)^{2}, Z_{k+1}=Z_{k}+V_{k} \cdot \sqrt{2} \sin \left(\frac{d_{k}}{2}\right) \cdot \cos \left(\frac{d_{k}}{2}\right)
$$

In Eq.9, $\left(\boldsymbol{X}_{k+1}, y_{k+1}, Z_{k+1}\right),\left(X_{k}, y_{k}, Z_{k}\right)$ are nodes positions in $k$ and $k+1$ moments. After all the anchor nodes' positions are updated, the algorithm will start the next round positioning.

Time delay in MANLFI algorithm calculated from 4 aspects: the time that anchor node broadcasts information, named $\boldsymbol{t}_{\text {bcast }}$; the time that the anchor node moves, named $\boldsymbol{t}_{\text {move }}$; the time that the unknown node responses the signal, named $\boldsymbol{t}_{\text {ret }}$, and the time that uses fuzzy information to locate node $\boldsymbol{t}_{\text {loca }}$, the total time can be calculated as Eq.10:

$$
T=t_{\text {bcast }}+t_{\text {ret }}+t_{\text {move }}+t_{\text {loca }}
$$

According to Eq.10, the total time of MANLFI, APIT-3D and Bounding cube can be described with Eq.11, Eq.12 and Eq.13:

$$
T_{\text {NMANLFI }}=\left(m\left(q^{2} \cdot A H-1\right)+n\right) \cdot N D \cdot t_{\text {bcast }}+\sum_{i=1}^{m} \sum_{k} d a_{(k, k+1)_{(i)}} \cdot t_{\text {move }}+(n-m) \cdot \mathrm{AH} \cdot \boldsymbol{t}_{\text {ret }}+\frac{m}{2} \cdot(n-m) \boldsymbol{t}_{\text {loca }}
$$




$$
\begin{aligned}
& T_{\text {APIT-3D }}=\left(m\left(\left(q^{4}+3 \cdot q\right) \cdot A H-1\right)+n\right) \cdot N D \cdot t_{\text {bcast }}+m \cdot A H \cdot t_{\text {ret }}+\frac{m}{4} \cdot(n-m) \cdot t_{\text {loca }} \\
& T_{\text {Bounding cube }}=\left[n+m \cdot\left(\left(q^{3}+5 \cdot q\right) \cdot A H-1\right)\right] \cdot t_{\text {bcast }}+m \cdot A H \cdot t_{\text {ret }}+\frac{m}{3} \cdot(n-m) \cdot t_{\text {loca }}
\end{aligned}
$$

The result of Eq.12minus Eq.11 is described as Eq.14:

$$
\begin{aligned}
T_{\text {APIT-3D }}-T_{\text {MANLFI }}= & \left(q^{4}+3 \cdot q-q^{2}\right) \cdot A H \cdot N D \cdot t_{\text {bcast }}-\sum_{i=1}^{m} \sum_{k} d a_{(k, k+1)_{(i)}} \cdot t_{\text {move }} \\
& +(2 m-n) \cdot A H \cdot t_{\text {ret }}-\frac{m}{4}(n-m) \cdot t_{\text {loca }}
\end{aligned}
$$

The result of Eq.13 minus Eq.11 is described as Eq.15:

$$
\begin{aligned}
T_{\text {Bounding cube }}-T_{\text {MANLFI }}= & \left(q^{3}+5 \cdot q-q^{2}\right) \cdot A H \cdot N D \cdot t_{\text {bcast }}-\sum_{i=1}^{m} \sum_{k} d a_{(k, k+1)_{(i)}} \cdot t_{\text {move }} \\
& +(2 m-n) \cdot A H \cdot t_{\text {ret }}-\frac{m}{6}(n-m) \cdot t_{\text {loca }}
\end{aligned}
$$

$q>1$, the power of $\mathcal{Q}$ is increased with exponential, and $t_{\text {bcast }} \gg t_{\text {move }}, t_{\text {bcast }} \gg t_{\text {loca }}$, so the items with $t_{\text {move }}, t_{\text {loca }}$ can be ignored, so the eventual results of comparison are described as Eq.16 and Eq.17.

$$
\begin{aligned}
& T_{\text {APIT-3D }}-T_{\text {MAIFF }} \approx m\left(q^{4}+3 \cdot q-q^{2}\right) \cdot N D \cdot t_{\text {bcast }} \\
& T_{\text {Bounding a ube }}-T_{\text {MANLFI }} \approx m\left(q^{3}+2 \cdot q-q^{2}\right) \cdot N D \cdot t_{\text {bcast }}
\end{aligned}
$$

From the above analysis, it can be seen obviously that the results of Eq.16 and Eq.17 are both greater than 0. In other words, the time delay of MANLFI is less than APIT-3D and Bounding cube.

\section{Simulation and Analyze}

In this section, the MATLAB programming environment is used to compare the performances of MANLFI, APIT-3D and Bounding cube, such as coverage ratio, error ratio and time delay . Assume the wireless sensor network deployed within the cube area of $1000 \times 1000 \times 100 \mathrm{~m}^{3}$ and sensor nodes are randomly deployed.The simulation parameters are shown in Table1.

Table.1The parameters in simulation

\begin{tabular}{cc}
\hline Parameters: & values: \\
\hline Total number of nodes $n$ & 200 \\
Node communication radius $\boldsymbol{r}_{c}$ & $10 \mathrm{~m}$ \\
Node sensing radius $\boldsymbol{r}_{s}$ & $5 \mathrm{~m}$ \\
Time of anchor nodes broadcast signal $\boldsymbol{t}_{\text {bcast }}$ & $10 \mathrm{~ms}$ \\
Time of unknown node return information $\boldsymbol{t}_{\text {ret }}$ & $10 \mathrm{~ms}$ \\
Time of using fuzzy information to calculate $\boldsymbol{t}_{\text {loca }}$ & $5 \mathrm{~ms}$ \\
Time of moving the anchor node $\boldsymbol{t}_{\text {move }}$ & $2 \mathrm{~ms} / \mathrm{m}$ \\
parameter $\boldsymbol{p}$ & 0.4 \\
parameter $\boldsymbol{q}$ & 5 \\
\hline
\end{tabular}

When anchor nodes occupy different proportions, the positioning error $\gamma$ of these three algorithms are compared. $\gamma$ can be calculated with $\gamma=\left(n-n^{\prime}\right) / n, n$ is the total number of unknown nodes, $n$ is the number of nodes that has been located. The comparison shown as Fig.2: 


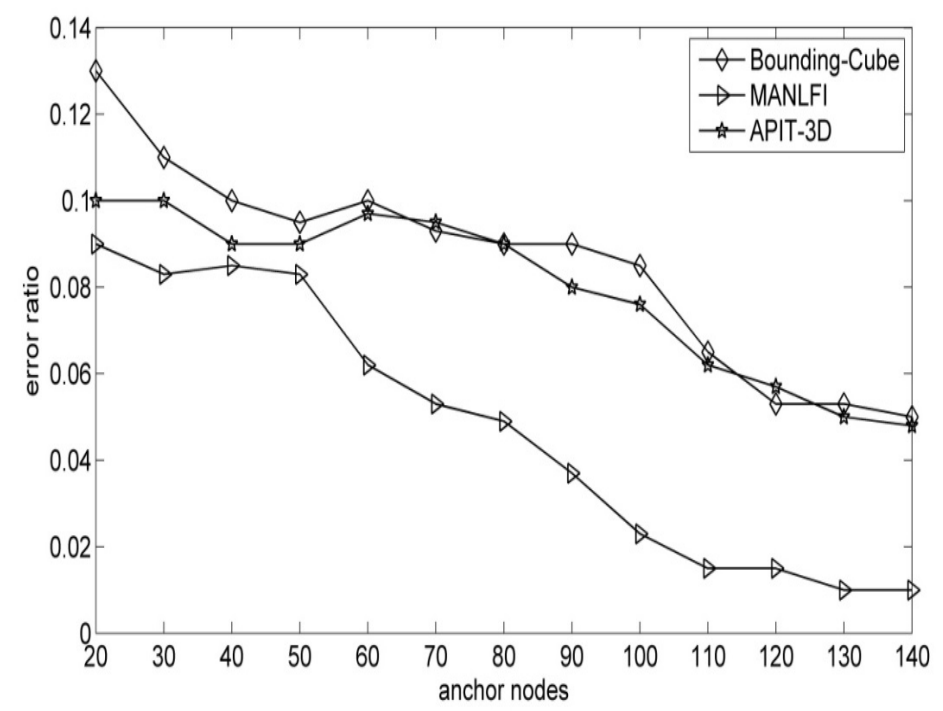

Fig.2. Comparisons of the locating error ratio

We can obviously see from the results of Fig.2 that the result is fully consistent with the forecast before simulation. In different proportions of anchor nodes, MANLFI has the lowest localization error compared with APIT-3D and Bounding cube. Especially when the anchor node is in a half number of all nodes, the positioning error will greatly reduce. This is owing to MANLFI based on mobile anchor node's fuzzy information to locate node. And after locating, using coordinates' average values to fix the position. After the comparison, we concluded that the positioning accuracy of MANLFI is higher.

When node is in different communication radius, the coverage rate of three algorithms are compared. The coverage ratio is defined as ratio that the volume has been covered with the total volume in space. The comparison shows as follows Fig.3.

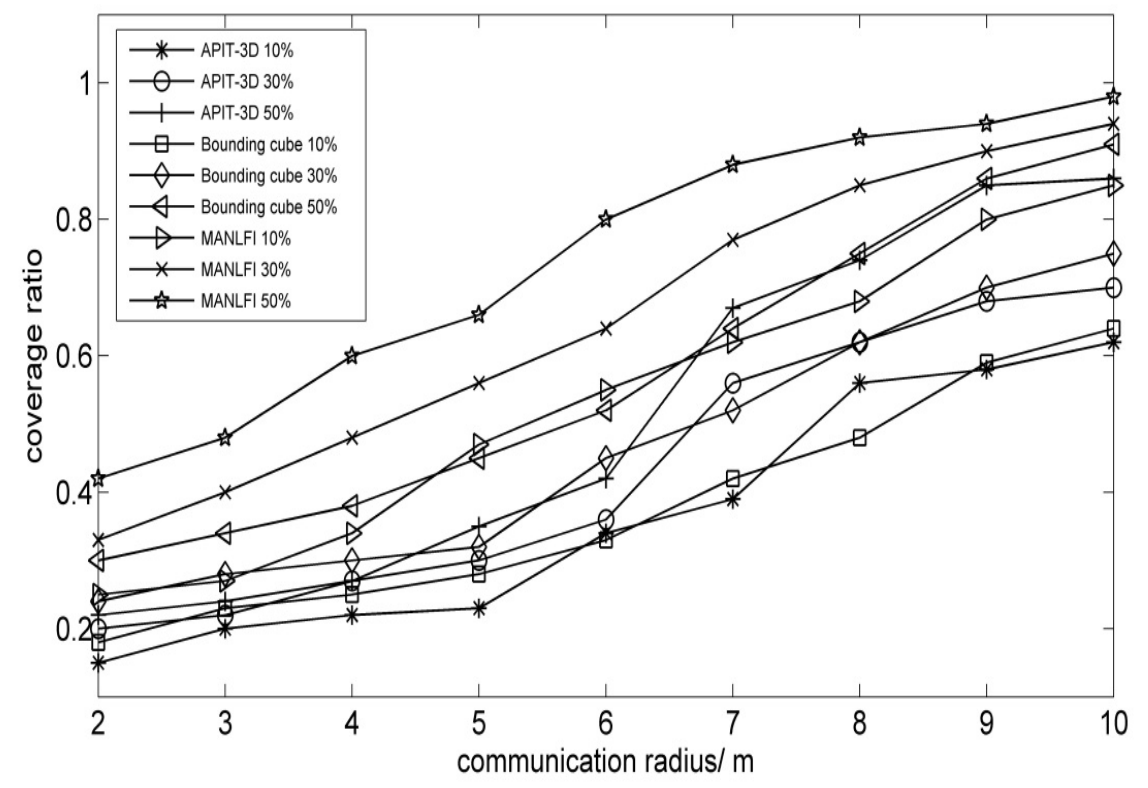

Fig.3. Coverage rate compared with different node's communication radius.

The coverage ratio is the key indicator value of the effectiveness. If the value is too low, it will seriously affect the application of the algorithm. From the simulation results in Fig.3, we can obviously see, with the communication radius increased, the coverage rate increased in each algorithm. All of these can be explained as when the communication radius increased, the perceived distance also increased. Especially in MANLFI, the anchor node can move and it will move to a suitable position before locating other node. When the positions of any nodes are fixed, they can 
locate other nodes but their positions will not change with the algorithm goes. So the coverage rate of MANLFI is greater than the other two algorithms.

The time delay comparison of MANLFI, APIT-3D and Bounding cube follows as Fig.4.
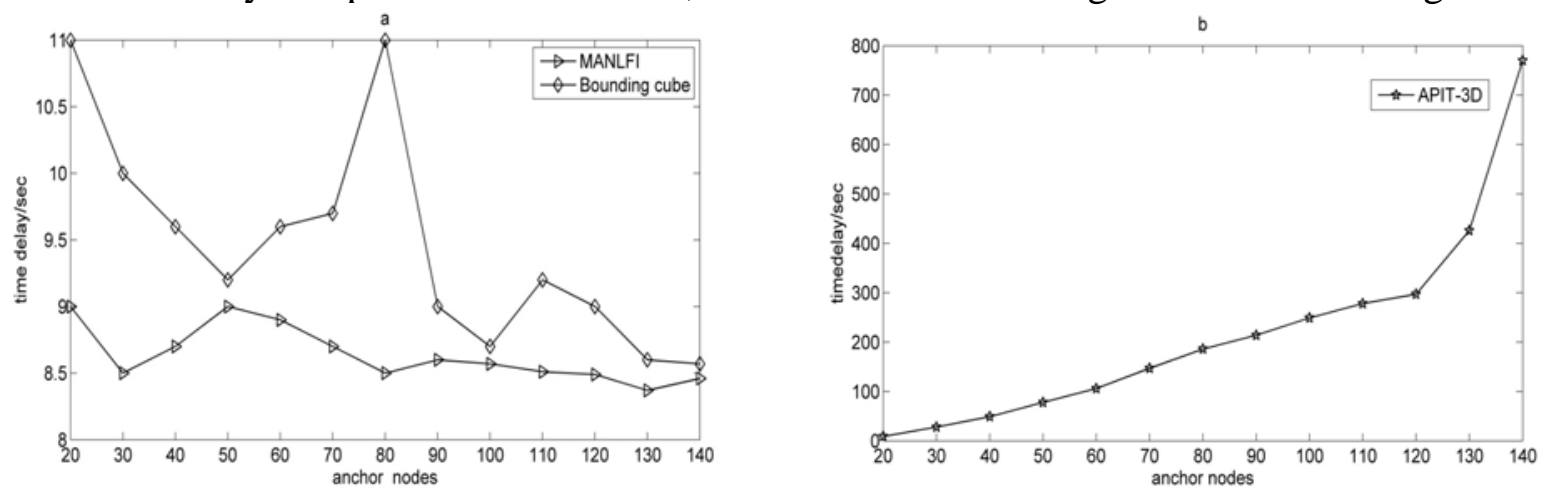

Fig.4. Comparisons of time delay

From the simulation results, we can see the range of time delay is fully consistent with our analysis before simulation. With anchor nodes occupying different proportions, we can see the time delay of these three algorithms is various, and the total trends are different. From Fig.4(b), we can find that the time delay of APIT-3D is increased with exponential trend. In Fig.4(a), the various trends of time delay in Bounding cube are uncertain and the trends of time delay in MANLFI tend to be a stable value and is the lowest value compared with APIT-3D and Bounding cube.

\section{Conclusions}

Both Bounding cube algorithm and APIT-3D algorithm have problems of low coverage ratio and low position accuracy. Through detailed analysis of the discrete network model, this paper presents an algorithm named MANLFI, based on mobile anchor nodes fuzzy information, to solve the nodes positioning problem in three-dimensional space. The knowledge of fuzzy information in three-dimensional space is introduced. In MANLFI algorithm, the anchor nodes can move constantly and the nodes will act as static anchor nodes to locate other nodes after being located. With simulation experiments, we analyzed and compared MANLFI with APIT-3D and Bounding cube from the aspects of positioning error ratio, coverage ratio, time delay. Experimental results show that MANLFI algorithm has greatly improved the coverage of the algorithm and reduced the positioning error. When the number of anchor nodes is less and the average connectivity is low in the network, compared with other traditional and improved algorithms, the algorithm proposed in this paper will significantly increase the coverage ratio as well as in ensuring network connectivity, MANLFI algorithm improves the position accuracy and greatly enhances the usefulness of the algorithm. So we can draw a conclusion that the MANLFI algorithm is more efficient in positioning and is in better performance compared with APIT-3D and Bounding cube.

\section{Acknowledgements}

This work was financially supported by The National Natural Science Fund (61363059) and Young Teachers' Scientific Research Ability Promotion Program of Northwest Normal University (NWNU-LKQN-13-24).

\section{References}

[1] Yan Zhou,Bao Wen,Jianxun Li.Closer Nodes Weighted Centroid Localization for Wireless Sensor Networks[J]. Computer Engineering and Applications, 2012,48(1):88-93.( In Chinese)

[2] Aitzaz Ahmad,Erchin Serpedin,Hazem Nounou, Mohamed Nounou.Joint Node Localization and Time-Varying Clock Synchronization in Wireless Sensor Networks. IEEE Transactions on Wireless Communications,2013,12(10). 
[3] Andrea Gasparri, Federica Pascucci, Giovanni Ulivi.A Distributed Extended Information Filter for Self-Localization in Sensor Networks. IEEE International Symposium on Personal, Indoor and Mobile Radio Communications.2008,19(1): 1774-1778.

[4] Mohamed K. Watfa, Sesh Commuri.Self Organization of Sensor Networks for Energy-Efficient Border Coverage[J]. Journal of Communications and Networks, 2009,11(1): 57-71.

[5] Raju Dutta, Shishir Gupta, Mukul.DAS.Power Consumption and Maxinizing Network Lifetime during Communication of Sensor Node in WSN[J]. Procedia Technology,2012:158-162.

[6] Hady S.AbdelSalam,Stephan Olariu,Syed R.Rizvi.Tiling-Based Localization Scheme for Sensor Networks using A Single Beacon.IEEE Global Telecommunications Conference, 2008,27(1) :790-794

[7] Congfei Tang, Li Peng.Competition Location of Anchor Nodes in Omni-Directional Vision Network[J]. Computer Engineering and Applications, 2014,50(10):147-151.( In Chinese)

[8] Dawood Al-Abri, Janise McNair.Improving Localization Accuracy in Wireless Sensor Networks using Location Verification Feedback.IEEE Military Communications Conference.2007(10):2296-2302.

[9] Mohamed Abid, Soumaya Cherkaoui.3D Compressive Sensing for Nodes Localization in WSNs Based On RSS. IEEE International Conference on Communications, 2012,35(1).

[10] Huadong Ma, Xi Zhang, Anlong Ming.A Coverage-Enhancing Method for 3D Directional Sensor Networks. IEEE International Conference on Computer Communications,2009,28(1): 2791-2795.

[11] Vijay K. Chaurasiya,Neeraj Jain, G.C. Nandi.A Novel Distance Estimation Approach for 3D Localization in Wireless Sensor Network using Multi-Dimensional Scaling[J].Information Fusion,2014,15:5-18.

[12] Zhendong Zeng.Study of Bounding Box Positioning algorithm Based on Wireless Sensor Discrete Network Model[J].Computer Applications and Software.2013,30 (12):194-257. (In Chinese)

[13] Dan Zuo, Sheng Xiao, Xinhai Lei.An Algorithm about Mobile Beacon Node Localization Based on Improved Bounding Box[J]. Command Control \& Simulation,2012,34(1):53-56.( In Chinese)

[14] Juan Li, Ke Wang, Changgang Lu.Bounding cube: An Algorithm for Wireless Sensor Network Node Localization in Three-Dimensional[J].Ocean University of China,2009,39(6):1265-1268.( In Chinese)

[15] Yunjian Xu, Aiyin Guo.A Research about Mobile Agent Routing Algorithm Based on APIT In Three-Dimensional[J]. Application Research of Computers, 2010,27(6):2246-2253.( In Chinese)

[16] Biao Han, Changbiao Xu, Hai Yuan, Lei Peng.Improved APIT Positioning Algorithm in Wireless Sensor Network[J]. Computer Engineering and Applications, 2008,44(4): 122- 124.( In Chinese)

[17] Yongjun Chen, Gang Xu, Xingju Zhou.Location Method of Minimum Number Communication Node for Wireless Sensor Networks[J].Computer Engineering and Applications,2014,50(5):55-59. (In Chinese)

[18] Dimitrios Zorbas,Tahiry Razafind Ralambo. Prolonging Network Lifetime under Probabilistic Target Coverage in Wireless Mobile Sensor Networks[J]. Computer Communications,2013:1039-1053.

[19] Chunhan Lin,Huangchen Lee,Chungta King.Periphery Deployment for Wireless Sensor Systems with Guaranteed Coverage Percentage[J].The Journal of Systems and Software,2011:763-774.

[20] Ze Wanga,Yunlong Wangaauthor Vitae,Maode Mabauthor Vitae,Jigang Wu.Efficient Localization for Mobile Sensor Networks Based on Constraint Rules Optimized Monte Carlo Method[J].Computer Networks, 2013,57(14):2788-2801.

[21] Dong-Kyu Lee,Tae-Hyon Kim,Seol-Young Jeong,Soon-Ju Kang.A Three-Tier Middleware Architecture Supporting Bidirectional Location Tracking of Numerous Mobile Nodes under Legacy WSN Environment[J]. Journal of Systems Architecture,2011: 735-748.

[22] Sudhir Kumar,Vatsal Sharan, Rajesh Hegde.Energy Efficient Optimal Node-Source Localization using Mobile Beacon in Ad-Hoc Sensor Networks.IEEE Global Telecommunications Conference, 2013,32(1).

[23] D.F.Larios,J.Barbancho,F.J.Molina,C.Leon.LIS:Localization Based on An Intelligent Distributed Fuzzy System Applied to A WSN[J].Ad Hoc Networks,2012:604-622.

[24] Mao Song,Zhao Cheng-Lin.Unequal Clustering Algorithm for WSN Based on Fuzzy Logic and Improved ACO[J]. Science Driect, 2011,18(6): 89-97.

[25] Raju Dutta,Sajal Saha,Asish K.Mukhopadhyay.Tracking Hetergeneous Dynamic Sensor Node Using Fuzzy Logic to Prolong System Lifetime in WSN[J]. Procedia Engineering,2012:522-527.

[26] Ben-Jye Chang,Jia-Bin Peng,Ying-Hsin Liang.Minimizing Transmission Delay and Deployment Cost for Sensors Placement in Sparse Wireless Sensor Networks.IEEE Wireless Communications and Networking Conference,2007,3: 2588-2592. 
[27] Massimo Vecchio,Roberto Lopez-Valcarce,Francesco Marcelloni.A Two-Objective Evolutionary Approach Based on Topological Constraints for Node Localization in Wireless Sensor Networks[J]. Applied Soft Computing, 2012,(7):1891-1901.

[28] F. Daneshgaran,M. Laddomada,M. Mondin.Connection between System Parameters and Localization Probability in Network of Randomly Distributed Nodes. IEEE Transactions on Wireless Communications,2007,6(12):4383-4389.

[29] Liang Dong.Cooperative Network Localization via Node Velocity Estimation. IEEE Wireless Communications and Networking Conference, 2009,10(1):2231-2236. 